\title{
Short-term monocular patching boosts the patched eye's response in visual cortex
}

\author{
Jiawei Zhou ${ }^{\mathrm{a}, 1}$, Daniel H. Baker ${ }^{\mathrm{b}, 1}$, Mathieu Simard ${ }^{\mathrm{c}}$, Dave Saint-Amour ${ }^{\mathrm{c}}$ and Robert F. Hess ${ }^{\mathrm{a}, *}$ \\ ${ }^{a}$ McGill Vision Research, Department of Ophthalmology, McGill University, Montréal, Canada \\ ${ }^{\mathrm{b}}$ Department of Psychology, University of York, Heslington, York, UK \\ ${ }^{\mathrm{c}}$ Department of Psychology, Université du Québec à Montréal, Montréal, Canada
}

\begin{abstract}
.
Purpose: Several recent studies have demonstrated that following short-term monocular deprivation in normal adults, the patched eye, rather than the unpatched eye, becomes stronger in subsequent binocular viewing. However, little is known about the site and nature of the underlying processes. In this study, we examine the underlying mechanisms by measuring steady-state visual evoked potentials (SSVEPs) as an index of the neural contrast response in early visual areas.

Methods: The experiment consisted of three consecutive stages: a pre-patching EEG recording (14 minutes), a monocular patching stage (2.5 hours) and a post-patching EEG recording (14 minutes; started immediately after the removal of the patch). During the patching stage, a diffuser (transmits light but not pattern) was placed in front of one randomly selected eye. During the EEG recording stage, contrast response functions for each eye were measured.

Results: The neural responses from the patched eye increased after the removal of the patch, whilst the responses from the unpatched eye remained the same. Such phenomena occurred under both monocular and dichoptic viewing conditions.

Conclusions: We interpret this eye dominance plasticity in adult human visual cortex as homeostatic intrinsic plasticity regulated by an increase of contrast-gain in the patched eye.
\end{abstract}

Keywords: Monocular patching, eye dominance plasticity, intrinsic plasticity, contrast-gain, steady-state visual evoked potentials, visual cortex

\section{Introduction}

The visual system is capable of being modified by visual experience, a property known as visual plasticity. Following the pioneering studies of Torsten Wiesel and David Hubel in identifying ocular dominance columns in visual cortex (Hubel \& Wiesel, 1959, 1962), experience-dependent eye dominance modification has become the most extensively studied form of neural plasticity. Previously, it has been shown that three days of monocular deprivation in juvenile animals (including mice, kittens and monkeys) shifts

\footnotetext{
${ }^{1}$ Co-first authors.

*Corresponding author: Robert F. Hess, McGill Vision Research, Department of Ophthalmology, McGill University, Montréal, H3A 1A1, Canada. Tel.: +1 514934 1934/Ext. 35306; Fax: +1 514843 1691; E-mail: robert.hess@mcgill.ca.
}

ocular dominance toward the nondeprived eye (i.e. the nondeprived eye becomes more dominant). These procedures were not effective in adult animals, indicating a developmental time window (critical period) for plasticity (Frenkel \& Bear, 2004; Hubel \& Wiesel, 1970; Hubel et al., 1977). There is also emerging evidence to show that long periods (longer than five days) of monocular deprivation (Sawtell et al., 2003) or shortterm monocular deprivation (three days) preceded by 10-day binocular visual deprivation (He et al., 2006) can also induce ocular dominance shifts toward the nondeprived eye in adult animals.

On the other hand, several recent studies have demonstrated that a degree of eye dominance plasticity occurs in adult humans after one eye is patched for a relatively short period of time (as little as 2.5 hours). Over these timescales, the patched eye, rather than the unpatched 
eye, becomes stronger in subsequent binocular viewing (Lunghi et al., 2011, 2013; Zhou, Clavagnier, et al., 2013; Zhou, Thompson, et al., 2013). These findings in human adults are not consistent with the previous reports based on animal studies possibly because of the very different time scales of deprivation. However, little is known about the site and nature of the underlying processes in human adults. In this study, we examine the mechanisms underlying this eye dominance plasticity in human adults by measuring steady-state visual evoked potentials (SSVEPs) as an index of the neural response to contrast of early visual areas. Unlike previous studies that typically measured responses at only a single contrast level (Bagolini et al., 1994; Johansson \& Jakobsson, 2000, 2006), we measured contrast response functions for each eye (Baker \& Vilidaite, 2014; Tsai et al., 2012) to obtain a better estimate of the contrastdependence of the patching-induced changes. We show that patching one eye with a diffuser for 2.5 hours in adult humans increases the neural response to stimuli in the patched eye, whilst the responses from the unpatched eye remain the same. Such phenomena occur under both monocular and dichoptic viewing conditions. These results could be explained by a homeostatic intrinsic plasticity that regulates an increase of contrastgain in the patched eye.

\section{Methods}

\subsection{Observers}

Twelve adults (age: $27.7 \pm 6.4$ years old, four females) with normal or corrected to normal vision participated to this study. Seven observers were tested in Montreal, and five in York. Observers wore their normal optical correction if required. A written informed consent was obtained from each of them before the start of the test. This study complied with the Declaration of Helsinki and was approved by the Institutional Review Boards of McGill University and the ethics committee of the Department of Psychology at the University of York. The methods were carried out in accordance with the approved guidelines.

\subsection{Apparatus}

All stimuli were generated and controlled by a PC running Matlab (MathWorks, Natick, MA) with PsychTool Box 3.0.9 extensions (Brainard, 1997; Pelli, 1997). Head-mounted goggles, Z800 pro gog- gles (eMagin Corp., Washington, DC) or Oculus Rift DK2 (Oculus VR, California) were used to dichoptically present images to the two eyes. The Z800 pro goggles (used in Montreal) were driven by a DualHead2Go display adaptor (Matrox Electronic Systems Ltd., Montreal, Canada) and had a simulated viewing distance of $3.6 \mathrm{~m}$, a spatial resolution of $800 \times 600$, a refresh rate of $60 \mathrm{~Hz}$ and a mean luminance of $60 \mathrm{~cd} / \mathrm{m}^{2}$ in each eye. The Oculus Rift DK2 goggles (used in York) had a spatial resolution of $960 \times 1080$, a refresh rate of $60 \mathrm{~Hz}$ and a mean luminance of $22 \mathrm{~cd} / \mathrm{m}^{2}$ in each eye. The mini-OLED screens of the goggles are linear in luminance response and do not require Gamma correction (Black et al., 2011).

We recorded EEG signals using $\mathrm{Ag}-\mathrm{AgCl}$ electrodes located at the occipital pole $(\mathrm{Oz}$ site), referenced to the vertex $(C z)$. A ground electrode was placed on the forehead. Electrode impedances were kept below $10 \mathrm{k} \Omega$, and produced clear signals with no obvious artefacts. The signals were amplified and digitised at $1 \mathrm{kHz}$ using a BrainAmp (BrainProducts $\mathrm{GmbH}$, Germany) and saved for offline analysis in Matlab. The display system was synchronized with the recording computer using an Arduino-based trigger device. We Fourier transformed the EEG data from each trial, and took the amplitude at the target and mask frequencies as our dependent variable.

\subsection{Design}

The experiment consisted of three consecutive stages: a pre-patching EEG recording (14 minutes), a monocular patching stage (2.5 hours) and a post-patching EEG recording (14 minutes; started immediately after the removal of the patch). During the patching stage, a transparent patch (also called a diffuser, which transmits light with $80 \%$ light transmission but not pattern - for instance, it was not possible to count fingers at a distance of $10 \mathrm{~cm}$ ) covered one randomly selected eye. Observers were permitted to carry out normal activities whilst patched, such as using a computer, reading or walking around. The positions of the electrodes were marked before the start of testing to make sure that identical positions were recorded from before and after patching.

\subsection{Stimuli}

Stimuli were patches of static binary noise windowed by a raised cosine envelope to subtend 3 degrees 
(a) Mask

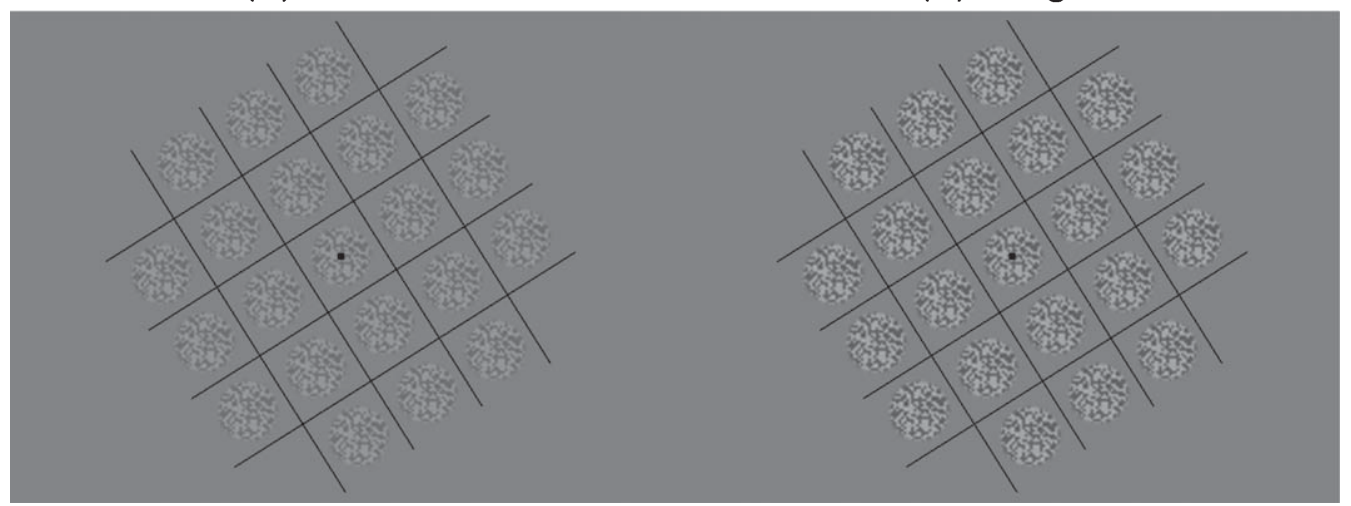

Fig. 1. Example stimuli used in experiments. Mask (a) and target (b) were patches of static white noise windowed by a raised cosine envelope. The patches were tiled in a $5 \times 5$ grid, surrounded by a series of orthogonal lines to aid binocular fusion. The orientation of the grids was varied randomly from trial to trial to minimise local adaptation.

of visual angle. The patches were tiled in a $5 \times 5$ grid, with the corner patches removed to avoid cropping at some orientations (see Fig. 1). To promote binocular fusion, a series of orthogonal lines crossed the display in between each patch. The orientation of the grid and placement of the patches was rotated by a random amount on each trial to minimise local contrast adaptation.

Target stimuli had maximum RMS contrasts of $3.2 \%$ $(10 \mathrm{~dB}), 12.5 \%(22 \mathrm{~dB})$ and $50 \%(34 \mathrm{~dB})$. Mask stimuli had a fixed RMS contrast of $26 \%$ ( $28 \mathrm{~dB})$. Target stimuli flickered at $10 \mathrm{~Hz}$ and mask stimuli flickered at $12 \mathrm{~Hz}$. The flicker was sinusoidal on/off flicker (e.g., the contrast varied from 0 to $100 \%$ of the maximum, and did not reverse the phase polarity of the stimuli).

\subsection{Procedure for EEG recording}

Observers were seated in a shielded room wearing the display goggles and EEG electrodes. Target stimuli were shown to either the left or right eye at a range of contrasts, for trials of 11 seconds with inter-trial intervals of 3 seconds. On some trials, the non-target eye viewed a blank screen showing only the fusion grid and a central fixation point. On the remainder of trials, a dichoptic mask was shown to the nontarget eye. This was spatially identical to the target, but flickered at a different temporal frequency. We used a dichoptic-masking paradigm because our previous psychophysics indicated that the patching effect was binocular in nature. This arrangement enabled us to measure the patching effect in different dichoptic- viewing conditions in the same session. In principle, improvements in sensitivity in one eye could result from an increase in gain for that eye, or a decrease in suppression from the other eye - the inclusion of a dichoptic condition allowed us to distinguish these possibilities.

There was no task during trials - observers were instructed to attend to the central fixation point and asked to avoid blinking during stimulus presentation. In total, there were 2 eyes $\times 2$ masking states (i.e., with/without mask) $\times 3$ target contrasts $=12$ conditions in the experiment. They were randomly presented in different trials. Each condition was repeated 5 times. Thus the test lasted 14 minutes in total.

\section{Results}

We averaged contrast response functions across our participants for the four dichoptic-viewing conditions (Fig. 2a), as shown in Fig. 2b-i. Before patching, the amplitude at the target frequency $(10 \mathrm{~Hz})$ increased monotonically as the target contrast increased (dashed lines and unfilled square symbols in Fig. 2b,c). A similar pattern was observed when a $12 \mathrm{~Hz}$ mask was presented simultaneously to the other eye (dashed lines and unfilled square symbols in Fig. 2d,e). The amplitude at the mask frequency $(12 \mathrm{~Hz})$ was at the noise level when no mask (i.e., blank) was shown (Fig. 2f,g). However, a strong response was found when the $12 \mathrm{~Hz}$ mask was presented (Fig. 2h,i) and the response declined slightly as the target contrast 
(a)
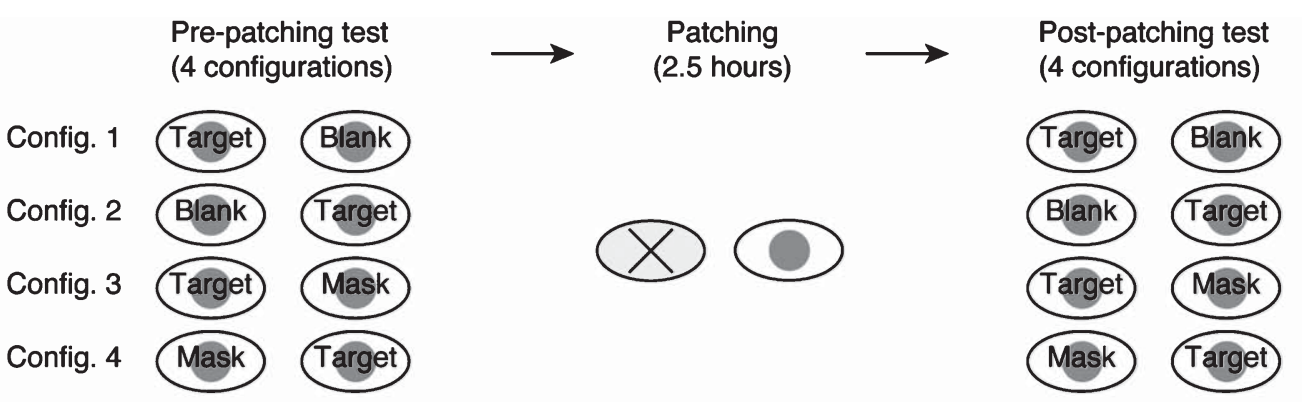

Target contrast (\%)

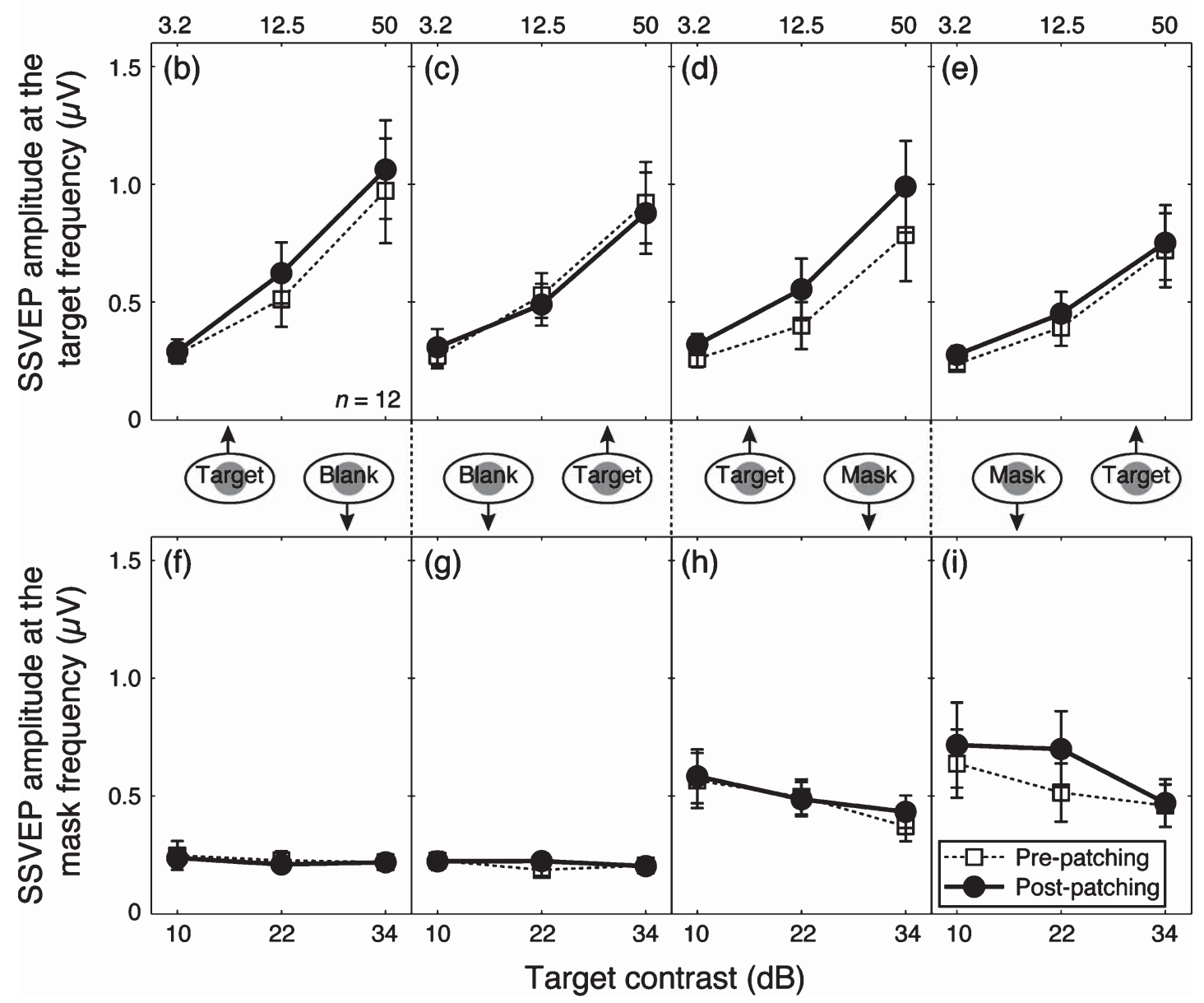

Fig. 2. Contrast response functions at the target and mask frequencies. (a) The procedure of the experiment. Contrast response functions for the target frequency and the mask frequency were tested at four configurations before and after the 2.5-hour patching stage. Panels (b-e) show the results for the target frequency and $(\mathrm{e}-\mathrm{h})$ show the results for the mask frequency. The four dichoptic conditions are listed in separate columns, in particular, (b) and (f) refer to the condition in which the previously patched eye saw the target and there was no mask in the unpatched eye; (c) and (g) refer to the condition in which the unpatched eye saw the target and there was no mask in the previously patched eye; (d) and (h) refer to the condition in which the previously patched eye saw the target and the unpatched eye saw the mask and (e) and (i) refer to the condition in which the unpatched eye saw the target and the previously patched eye saw the mask. In each panel, the pre-patching measures are presented as unfilled squares and dashed lines and the post-patching measures are presented as filled circles and solid lines. Error bars give \pm 1 standard error across observers $(n=12)$. 


\section{Target contrast (\%)}

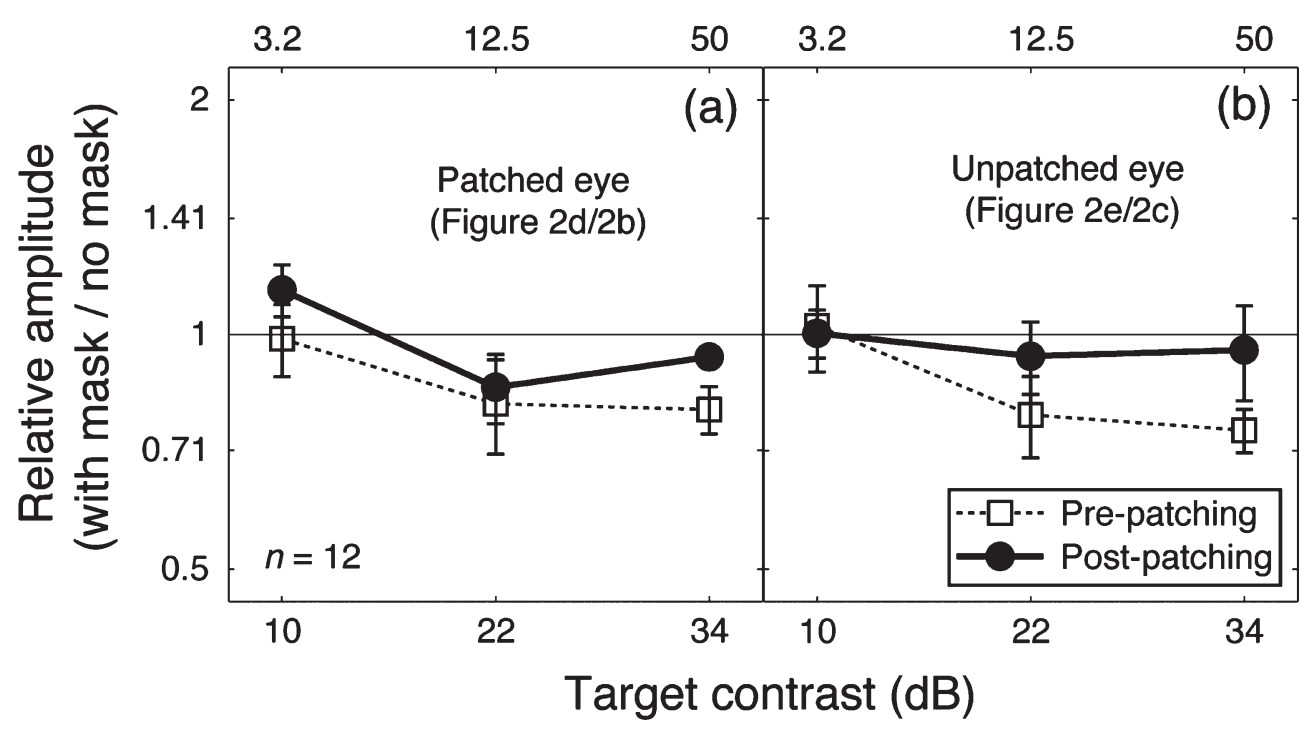

Fig. 3. The dichoptic masking effect on SSVEP amplitudes at the target frequency. Relative SSVEP amplitude (with mask/no mask) plotted against target contrast for the patched eye (a) and unpatched eye (b). Points lower than the middle identity line indicate dichoptic masking effects of the mask on SSVEP amplitudes at the target frequency. Error bars give \pm 1 standard error across observers $(n=12)$.

increased. These contrast gain control effects are consistent with previous work using similar techniques (Baker \& Vilidaite, 2014; Candy et al., 2001; Tsai et al., 2012).

Patching one eye for 2.5 hours increased the amplitude of the contrast response for that eye at the target frequency (solid lines and filled circles in Fig. 2b,d). The amplitude at the mask frequency also increased when the previously patched eye saw the mask (solid lines and filed circles in Fig. 2i). The amplitude in other conditions was unaffected by patching. We ran a 3-factor repeated-measures within-subject Analysis of Variance (ANOVA) for each eye, with patching (prepatching and post-patching, 2 levels), target contrast $(10,22$, and $34 \mathrm{~dB}, 3$ levels) and masking (with no mask and with mask, 2 levels) as within subject factors to statistically assess the patching effect on the two eyes' response at the target frequency. For the patched eye (results in Fig. $2 \mathrm{~b}$ and d), the patching effect was significant $(\mathrm{F}(1,11)=5.70, p=0.036)$, the masking effect was also significant $(\mathrm{F}(1,11)=12.48$, $p=0.005$ ); for the unpatched eye (results in Fig. $2 \mathrm{c}$ and e), patching had no significant effect $(\mathrm{F}(1,11)=0.21$, $p=0.65)$, whilst the masking effect was still significant $(\mathrm{F}(1,11)=15.73, p=0.002)$. To better show the dichoptic masking effect on SSVEP amplitudes at the target frequency, we calculated the relative amplitude (with mask/no mask) for the patched eye (i.e., results in Fig. 2d/results in Fig. 2b) and the unpatched eye (i.e., results in Fig. 2e/results in Fig. 2c), as shown in Fig. 3. The masking effect (i.e., relative amplitudes less than 1) was evident especially at high target contrasts for both eyes. Patching did not significantly change the masking effect for either eye (Fig. 3a, $\mathrm{F}(1,11)=1.40, p=0.26$; Fig. 3b, $\mathrm{F}(1,11)=1.77, p=0.21)$.

We also combined the results at both the target frequency and the mask frequency and ran a 3-factor repeated-measures within-subject ANOVA for each eye, with patching (2 levels), target contrast (3 levels) and dichoptic-viewing condition (the response to target in the monocular viewing, the response to target in the dichoptic viewing and the response to mask in the dichoptic viewing, 3 levels) as within subject factors, to statistically assess the patching effect on the two eyes' contrast response functions by evaluating the interaction between patching level and contrast level. We found that the patching effect on the contrast response functions was significant in the patched eye (Fig. 2b,d,i; $\mathrm{F}(2,22)=4.26, p=0.03$ ) but not significant in the unpatched eye (Fig. 2c,e,h; F(2,22) $=0.60$, $p=0.56)$. These results suggest that patching strengthened the patched eye's response, whilst the responses from the unpatched eye remained the same. 


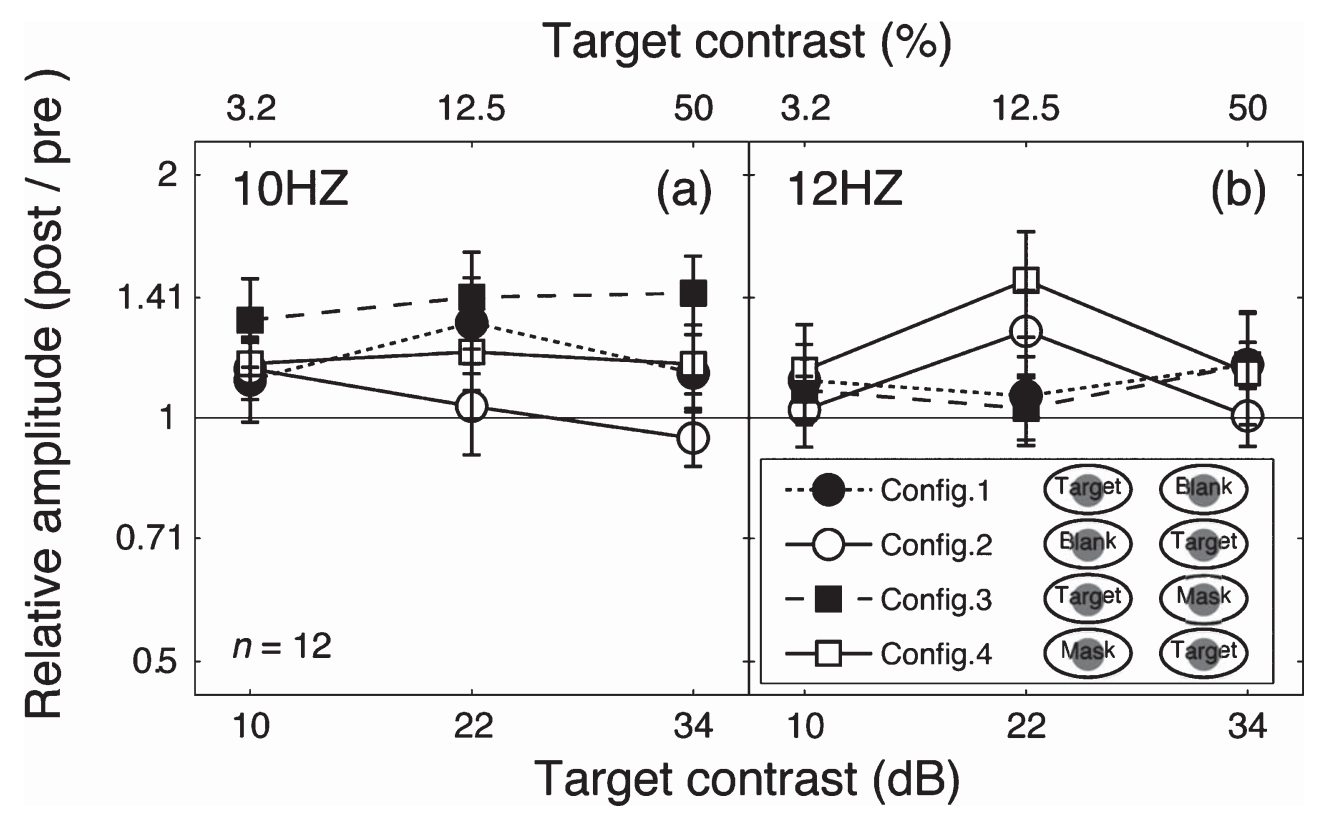

Fig. 4. Change of SSVEP amplitudes after patching. Relative SSVEP amplitude (Post/pre) plotted against target contrast for the four viewing conditions. Points above the middle identity line indicate increasing of response after 2.5 -hour patching. Error bars give \pm 1 standard error across observers $(n=12)$.

To further illustrate this finding, we calculated the relative amplitude (post-patching/pre-patching) for the target frequency (Fig. 4a) and the mask frequency (Fig. 4b). These data replicate the main features of the contrast response function changes in Fig. 2. Patching induced a strengthening effect in the patched eye's responses (filled squares and filled circles in Fig. 4a and unfilled squares in Fig. 4b), but no response change was observed for the unpatched eye (unfilled squares and unfilled circles in Fig. 4a and filled squares in Fig. 4b).

\section{Discussion}

Our data allow objective verification of a novel form of eye dominance plasticity in the adult human visual cortex. Previous behavioral findings in adult (Lunghi et al., 2011, 2013; Zhou, Clavagnier, et al., 2013; Zhou, Thompson, et al., 2013), have shown that monocular patching for a period as short as 2.5 hours strengthens the psychophysically measured sensitivity of the patched eye. Our data demonstrate that the contrast response of the visual cortex driven by the patched eye is increased in both monocular and dichoptic viewing conditions after 2.5 hours of monocular patching.

It is noteworthy that, unlike the bidirectional shifting of contrast response functions in the two eyes that was previously reported using behavioral testing (Zhou, Clavagnier, et al., 2013), here for the cortical SSVEP response, we only found a leftward shift of the patched eye's contrast response function with no clear change in the unpatched eye's response. This may be due to the sensitivity of the SSVEP approach since the psychophysically measured decrease in sensitivity of the unpatched eye is subtle, or it may be because the neural locus of the psychophysical effect is different from that of the SSVEP effects measured here.

The leftward-shift of contrast response functions for the patched eye provides a means of interpreting the previously reported behaviorally-measured improvement in sensitivity of the patched eye's response. A change in behavioral sensitivity could be the result of neural contrast response functions shifting vertically as a result of changes in neural noise and/or laterally due to changes in gain (see (Kwon et al., 2009)). Assuming the SSVEP response is a valid reflection of neural contrast responses, the present results, based on measures at three target contrast levels, suggest the sensitivity improvement of the patched eye due to short term patching could be a consequence of an increase in contrast-gain (or a reduction of suppression) and/or a reduction in neural noise. Such a change in the balance of excitation and suppression associated with binocular combination (Meese et al., 2006) is in good agreement 
with the idea of homeostatic intrinsic plasticity (Desai et al., 1999; Mrsic-Flogel et al., 2007; Ranson et al., 2012), for review, see (Turrigiano, 2011). In particular, it is possible that during the patching stage when neural responses corresponding to the patched eye's contribution to binocular combination are much reduced, neurons might respond by regulating their intrinsic properties to shift their input/output function to the left (increase the contrast gain or decrease interocular inhibition) in an effort to strengthen the patched eye's contribution to the binocular percept.

\section{Acknowledgments}

This study was supported by a Natural Sciences and Engineering Research Council of Canada grant (NSERC \#46528) to RFH, a Canadian Institutes of Health Research grant (CIHR-53346) to RFH, a RIMUHC Postdoctoral award to JZ, and FRSQ Vision Heath Research Network awards to DSA and RFH.

\section{References}

Bagolini, B., Falsini, B., Cermola, S., \& Porciatti, V. (1994). Binocular interactions and steady-state VEPs. A study in normal and defective binocular vision (Part II). Graefes Arch Clin Exp Ophthalmol, 232(12), 737-744.

Baker, D.H., \& Vilidaite, G. (2014). Broadband noise masks suppress neural responses to narrowband stimuli. Front Psychol, 5, 763, $1-9$.

Black, J.M., Thompson, B., Maehara, G., \& Hess, R.F. (2011). A compact clinical instrument for quantifying suppression. Optom Vis Sci, 88(2), 334-343.

Brainard, D.H. (1997). The psychophysics toolbox. Spatial Vision, $10(4), 433-436$.

Candy, T.R., Skoczenski, A.M., \& Norcia, A.M. (2001). Normalization models applied to orientation masking in the human infant. Journal of Neuroscience, 21(12), 4530-4541.

Desai, N.S., Rutherford, L.C., \& Turrigiano, G.G. (1999). Plasticity in the intrinsic excitability of cortical pyramidal neurons. Nature Neuroscience, 2(6), 515-520.

Frenkel, M.Y., \& Bear, M.F. (2004). How monocular deprivation shifts ocular dominance in visual cortex of young mice. Neuron, 44(6), 917-923.

He, H.-Y., Hodos, W., \& Quinlan, E.M. (2006). Visual deprivation reactivates rapid ocular dominance plasticity in adult visual cortex. J Neurosci, 26(11), 2951-2955.

Hubel, D.H., \& Wiesel, T.N. (1959). Receptive fields of single neurones in the cat's striate cortex. J Physiol, 148, 574-591.

Hubel, D.H., \& Wiesel, T.N. (1962). Receptive fields, binocular interaction and functional architecture in the cat's visual cortex. J Physiol, 160, 106-154
Hubel, D.H., \& Wiesel, T.N. (1970). The period of susceptibility to the physiological effects of unilateral eye closure in kittens. The Journal of Physiology, 206(2), 419-436.

Hubel, D.H., Wiesel, T.N., \& LeVay, S. (1977). Plasticity of ocular dominance columns in monkey striate cortex. Philos Trans $R$ Soc Lond B Biol Sci, 278(961), 377-409.

Johansson, B., \& Jakobsson, P. (2000). Fourier analysis of steadystate visual evoked potentials in subjects with normal and defective stereo vision. Documenta Ophthalmologica, 101(3), 233-246.

Johansson, B., \& Jakobsson, P. (2006). Fourier-analysed steady-state VEPs in pre-school children with and without normal binocularity. Documenta Ophthalmologica, 112(1), 13-22.

Kwon, M., Legge, G.E., Fang, F., Cheong, A.M., \& He, S. (2009). Adaptive changes in visual cortex following prolonged contrast reduction. Journal of Vision, 9(2), 20, 1-16.

Lunghi, C., Burr, D.C., \& Morrone, C. (2011). Brief periods of monocular deprivation disrupt ocular balance in human adult visual cortex. Current Biology, 21(14), R538-R539.

Lunghi, C., Burr, D.C., \& Morrone, M.C. (2013). Long-term effects of monocular deprivation revealed with binocular rivalry gratings modulated in luminance and in color. Journal of Vision, 13(6), 1, 1-15.

Meese, T.S., Georgeson, M.A., \& Baker, D.H. (2006). Binocular contrast vision at and above threshold. Journal of Vision, 6(11), 1224-1243.

Mrsic-Flogel, T.D., Hofer, S.B., Ohki, K., Reid, R.C., Bonhoeffer, T., \& Hubener, M. (2007). Homeostatic regulation of eye-specific responses in visual cortex during ocular dominance plasticity. Neuron, 54(6), 961-972.

Pelli, D.G. (1997). The VideoToolbox software for visual psychophysics: Transforming numbers into movies. Spatial Vision, 10(4), 437-442.

Ranson, A., Cheetham, C.E.J., Fox, K., \& Sengpiel, F. (2012). Homeostatic plasticity mechanisms are required for juvenile, but not adult, ocular dominance plasticity. Proceedings of the National Academy of Sciences, 109(4), 1311-1316.

Sawtell, N.B., Frenkel, M.Y., Philpot, B.D., Nakazawa, K., Tonegawa, S., \& Bear, M.F. (2003). NMDA receptor-dependent ocular dominance plasticity in adult visual cortex. Neuron, 38(6), 977-985.

Tsai, J.J., Wade, A.R., \& Norcia, A.M. (2012). Dynamics of normalization underlying masking in human visual cortex. The Journal of Neuroscience, 32(8), 2783-2789.

Turrigiano, G. (2011). Too many cooks? Intrinsic and synaptic homeostatic mechanisms in cortical circuit refinement. Annual Review of Neuroscience, 34(1), 89-103.

Zhou, J., Clavagnier, S., \& Hess, R.F. (2013). Short-term monocular deprivation strengthens the patched eye's contribution to binocular combination. Journal of Vision, 13(5), 12, 1-10.

Zhou, J., Thompson, B., \& Hess, R.F. (2013). A new form rapid binocular plasticity in adult with amblyopia. Scientific Reports, 3(2638), 1-5. 